\title{
Traduire
}

Une autre perspective sur r tr traduction

Revue française de la traduction

$226 \mid 2012$

Face au miroir

\section{Quand la langue rend la licorne toute chèvre}

\section{Shane Maloney}

Traducteur : Rose-Marie Vassallo et Dominique Durand-Fleischer

\section{(2) OpenEdition}

Journals

Édition électronique

URL : http://journals.openedition.org/traduire/142

DOI : $10.4000 /$ traduire. 142

ISSN : 2272-9992

Éditeur

Société française des traducteurs

Édition imprimée

Date de publication : 1 janvier 2012

Pagination : 45-50

ISBN : 039-773X

ISSN : 0395-773X

\section{Référence électronique}

Shane Maloney, "Quand la langue rend la licorne toute chèvre », Traduire [En ligne], 226 | 2012, mis en ligne le 04 février 2014, consulté le 09 octobre 2020. URL : http://journals.openedition.org/traduire/ 142 ; DOI : https://doi.org/10.4000/traduire.142 


\title{
Quand la langue rend la licorne toute chèvre
}

\author{
Shane Maloney \\ Traduction : Rose-Marie Vassallo et Dominique Durand-Fleischer
}

\section{NOTE DE L'ÉDITEUR}

Cet article est paru pour la première fois dans le bulletin Australian Author, vol. $36 \mathrm{n}^{\circ} 3$, décembre 2004. La version originale est consultable à l'adresse : http:// shanemaloney.com/articles-and-speeches/post/on-being-translated/

\section{NOTE DE L'AUTEUR}

Une fois votre roman traduit depuis l'anglais vernaculaire d'Australie, vous vous retrouvez tout Hyva Yritys, déclare l'écrivain Shane Maloney.

(Note des traductrices : le lecteur curieux et impatient trouvera à la fin du texte un petit lexique bilingue australien-français.)

1 La république des lettres a toujours été une communauté internationale. Et la plupart d'entre nous, faute d'avoir la chance d'être brillamment multilingues, n'accédons au trésor commun que par le biais de la traduction. C'est au travers d'un interprète que nous découvrons les mots des grandes figures de la littérature mondiale - Tolstoï, Boccace, Dumas, Kafka, sans parler de ce rigolo d'Argentin aveugle au nom quasi imprononçable. Lorsque nous lisons un livre traduit, nous savons implicitement que tout ne passe pas. Nous acceptons cette fatalité : quelque chose d'inhérent à l'original est perdu. Nous nous remettons entre les mains du traducteur et traçons notre sillon de lecteur. Si le texte enflamme notre imagination, sa provenance étrangère n'a pas à interférer. D'ailleurs, pour se sentir auteur à part entière, rien de tel que de se voir publié dans une langue étrangère. Car enfin, quoi de plus gratifiant que de retrouver ses propres écrits rendus littéralement illisibles par un parfait inconnu, dans un pays 
dont on ignore tout? À ce jour, j'ai été transmué en allemand, en français, en finnois, en japonais, en américain. Et je tiens de source sûre que le croate n'est pas loin.

2 Mais si j'ai la satisfaction de savoir qu'à Ingolstadt, Jyväskylä, Matsuyama, les lecteurs bénéficient enfin d'un accès direct à mon œuvre, chacun dans sa langue respective, j'avoue qu'une question me tarabuste : en matière de nuances, à quoi ont-ils droit au juste? En ont-ils pour leurs euros, leurs markaas, leurs yens? Leur parler local peut-il se plier aux exigences de mon style? Mes métaphores filent-elles? Mes envolées lyriques s'envolent-elles sur les ailes de la lyre? Et quid de mon louchebem, mon largonji, mes formules qui claquent, mes répliques pète-sec? En un mot, que vaut la traduction?

3 La traduction est un exercice notoirement épineux, après tout, et ses faiblesses intrinsèques sont déplorées depuis longtemps. Si l'on en croit Cervantès, la traduction est l'envers de la tapisserie. Comme il a probablement fait cette déclaration en espagnol, certaines subtilités ont pu se perdre en route. L'essentiel du propos est pourtant assez clair. Une traduction est beaucoup plus confuse que l'original, de nombreux fils mal noués s'en échappent et la licorne a finalement l'air d'une chèvre. Dans mon cas, le phénomène est aggravé par le fait que j'écris (ainsi que le mentionne mon éditeur allemand sur la page de titre) en anglais d'Australie. Et comme chacun sait, notre parler natal est un mode d'expression relativement obscur, si bien que je ne peux m'empêcher de me demander s'il est seulement possible de le rendre compréhensible dans une autre langue.

Mon initiation au monde de la traduction a commencé par un torrent de recommandations et de questions de la part de mon éditeur américain. Au début, ces remarques portaient sur des modifications mineures, juste un léger bricolage marginal. Il fallait adapter l'orthographe aux usages américains (-ise devenant -ize et colour devenant color). Les faux amis et les tournures peu familières devaient être gommés (du genre shoppingtrolley versus grocery cart et diary versus calendar). Et certains points de vocabulaire peu familiers exigeaient une clarification. Pourrais-je avoir l'amabilité de donner le sens et de fournir des termes de remplacement pour les expressions suivantes? Franger. Duco. Shoot through. Op shop. Furphy. Laminex. Ruckman. Fibro. A piece of piss. An unreconstructed Whitlamite. Ce n'est qu'après nous être livrés à ce ménage basique que nous sommes arrivés aux choses sérieuses, le nœud inter-culturel qui tenait tout le reste. L'usage américain exigeait de marcher sur un sidewalk et pas un footpath. "Allez vous faire foutre", ai-je déclaré, en ces termes ou en termes équivalents. Nous n'avons pas de sidewalks en Australie. Nous avons des footpaths. Et alors que je ne voyais aucune raison impérieuse de jouer les martyrs pour défendre le franger et le dunny, il n'était pas question que je permette à Murray Whelan de déambuler sur un putain de sidewalk américain. Tant qu'on y était, pourquoi pas lui faire manger des biscuits Oreo, boire de la Miller et voter républicain. En fin de compte une version mutuellement acceptable a été concoctée, avec juste assez de tournures australiennes pour que le lecteur américain moyen puisse les comprendre raisonnablement. Ils sont quand même plusieurs millions, a fait valoir mon éditeur américain avec enthousiasme.

5 Au moins les Américains m'avaient-ils consulté. Avec les Finlandais, je n'ai pas eu le moindre échange épistolaire. Vraisemblablement, Helsinki est correctement pourvu en Whitlamites impénitents sachant faire le distinguo entre franger et furfy. Ou peut-être mon traducteur s'est-il douté que mes connaissances de la branche finno-ougrienne de 
l'arbre des langues étaient, tout au mieux, ténues. Quel que soit le cas (et, à propos de cas, les esprits curieux seront heureux d'apprendre qu'il en existe quinze en finnois, dont le partitif, l'allatif, l'instructif et le comitatif), un livre en finnois a fait son apparition un beau jour dans ma boîte aux lettres. Tout ce que j'ai pu y déchiffrer, c'est mon nom. Et encore, parce qu'il figurait sur la couverture. Son titre? Hyvä Yritys. Ne me demandez pas ce que cela signifie, mais je trouve que ça claque bien, ça pète sec, et j'en déduis qu'ils ont trouvé le ton juste.

Quant à l'allemand, voilà bien une langue dont ma compréhension totalement nulle est. Mais le travail est absolument superbe. On peut s'en rendre compte rien qu'au poids. Par exemple la prédilection pour ces noms composés pour lesquels je ressens une tendresse particulière. Dès que le Kunstlerpech est arrivé, j'ai feuilleté les pages pour trouver des exemples. À ma grande joie, mes yeux ont été attirés immédiatement par le mot Satellitenavigationssystem. Apparemment, j'avais Schreiben auf Deutsch tout du long mais je ne l'avais pas réalisé. C'est quelque chose dont Diogenes Verlag AG aurait dû tenir compte quand ils ont traduit le titre Stiff par Weck mich, bevor du gehst. Quel qu'en soit le sens, cette expression ne claque pas du tout. C'est, comment dire, eine kleine schnapbereft. Mais peut-être que ces dernières années un effort conscient a été fait pour empêcher l'allemand de trop claquer. Peut-être que les voisins ont insisté.

7 Pour ce qui est du français, la collaboration (faute de terme meilleur) aura été plus active. Ne serait-ce que parce que, pour ma part, en raclant les fonds de tiroir, je rassemble suffisamment de parlez-vous pour suivre, en gros, mon texte en français. Ainsi, chaque fois qu'un de mes personnages entre dans un bistrot ${ }^{1}$ et commande une douzaine d'escargots et un verre de rouge, je me fais une idée assez claire de l'action. Cela dit, les petites subtilités comme le ton, le style me passent nettement au-dessus de la tête, or traduire en français ne se résume pas à malaxer la syntaxe. Un certain degré de haute cogitation est requis, comme j'ai pu m'en apercevoir. Non content de requérir des éclaircissements sur hoon, Paddle Pop, blue heeler et doona, mon traducteur $^{2}$ a également voulu savoir ce que j'entendais par bonking her ears off, living the life of Riley et being off with the pixies. Le temps de venir à bout de Clive James, de la rivalité Melbourne-Sidney et de la recette du witchetty-grub cappuccino, j'étais de force à tenir une chaire d'anthropologie sociétale des Antipodes à la Sorbonne. Aussie comme Autre.

Mais les contenus ne sont qu'une partie de l'histoire. Entre les mains françaises, les livres eux-mêmes passent de l'état d'objet physique à celui d'artefact culturel. Comme mes romans sont classés dans le vaste champ de la fiction avec intrigue criminelle, la logique cartésienne en a fait des romans policiers ${ }^{3}$. Ainsi, pour se conformer aux conventions du genre, sont-ils imprimés sur du mauvais papier bon marché et reliés entre deux couvertures jaune uni. Quand je feuillette un exemplaire, je peux presque sentir l'encre qui déteint et la colle qui s'en va.

On peut dire ce qu'on veut des Frenchies, ils savent traiter la littérature de gare avec le respect qu'elle mérite. Les Japonais, au contraire, demeurent impénétrables. Mon premier et unique contact avec mon traducteur japonais a eu lieu au lancement du livre. Nous avons échangé nos cartes de visite et signé nos exemplaires respectifs. La conversation est restée minimaliste. Pour vous dire la vérité, je ne suis pas sûr que M. Yakusha parle anglais, bien que je veuille croire qu'il le lit et l'écrit avec un haut degré de compétence. Concernant sa traduction, tout ce que je peux affirmer est qu'elle se lit de la fin du livre au début et de droite à gauche. Sans être entièrement conforme à l'original, cela représente sans doute une amélioration. De toute façon, le livre lui- 
même a été adapté à cette culture. Tout d'abord il a été miniaturisé, réduit à un format poche pour être plus facile à lire dans un métro bondé. Des publicités ont été insérées entre les pages pour faire la promotion de produits inconnus à des prix soldés. Et comme un kimono, le livre est entouré d'une obi. À cette occasion, j'aurais aimé échanger quelques explications avec Yakusha-san. Uniquement d'un point de vue professionnel, bien sûr, pour satisfaire ma curiosité linguistique. Certains personnages de mes livres, membres de la classe politique australienne, parlent un langage de corps de garde et je ne peux m'empêcher de me demander comment cela a été rendu en japonais, cette langue toute imprégnée de politesse. Même mes allusions pleines de tact ont dû représenter un énorme défi pour M. Yakusha. Par exemple, l'exclamation far canal! peut facilement poser problème à qui n'a pas immédiatement reconnu la transcription phonétique de la prononciation vernaculaire d'un juron populaire. Est-ce que par inadvertance il n'aurait pas été traduit "canal lointain » au lieu de «\%\&@\#*! ! ?

Remarquons qu'il n'est pas nécessaire d'aller très loin pour rencontrer ce genre de malentendu. On peut en trouver dans les endroits les plus inattendus. Dans les éditions britanniques de mes livres, le texte est exactement le même que celui de l'édition australienne. Seule la couverture change. Et voilà le hic. Le résumé qui figure sur la quatrième de couverture qualifie mon protagoniste de spin doctor. Dans le système politique britannique contemporain, c'est l'équivalent le plus proche de ce que nous appelons un conseiller ministériel. Malheureusement, ce n'est pas la description d'un métier. C'est un terme synonyme de malhonnêteté, de corruption et d'un manque total de principes et de sens moral. Franchement, les éditeurs auraient aussi bien fait de dire que mon héros est un parfait salaud.

Mais si ténues soient nos lignes de communication et si vifs les doutes qui m'habitent quant au résultat de leurs efforts, je tiens tous mes traducteurs dans la plus haute estime. Non seulement ils ont entrepris là une tâche qui dépasse de loin mes propres capacités, mais encore ils l'ont fait pour ainsi dire dans l'anonymat. Tout le boulot est pour eux, et c'est moi qui ai mon nom sur la couverture. Pour ce, je les salue bien bas, tous autant qu'ils sont, mes partenaires invisibles, mes co-auteurs. Avec mention spéciale pour ce type de Finlande. À lui, je dis : « Hyvä Yritys, vieux frère. J'adore ce que tu as fait avec les umlauts. "

\section{ANNEXES}

\section{Petites notes pour ignares pressés, voulant s'épargner une balade sur le Net}

Aussie : (terme familier, anglais d'Australie) n. 1. un Australien ; 2. Australie - adj : australien.

franger : (argot d'Australie) condom

Duco : peinture automobile (du nom du fabricant Dupont Company) 
op shop : opportunity shop, magasin d'articles d'occasion

$\mathbf{O z}$ : (terme familier, en anglais d'Australie) Australie

shoot through : partir

furphy : rumeur fausse ou invérifiable

Laminex : Formica

piece of piss : travail facile, du gâteau!

ruckman : terme tiré du football australien. Désigne un joueur clé, habituellement de grand gabarit, qui joue au centre et intercepte la balle en vol en sautant le plus haut possible. Équi- valent à un demi de mêlée.

Fibro : fibrociment

Dunny : (argot d'Australie) toilettes à l'extérieur sans eau courante

Hoon : hooligan

Paddle Pop : confiserie glacée à base de lait comme nos esquimaux

Blue Heeler : chien berger australien

doona : (marque déposée) couette garnie de duvet naturel ou synthétique.

Unreconstructed Whitlamite : (politique australienne des années quatre-vingts) terme péjoratif utilisé par les membres du cabinet Hawke-Keating pour désigner les autres membres du cabinet et du parti travailliste australien qui défendaient un point de vue en désaccord avec la ligne de libéralisme orthodoxe suivie par les personnes clés du gouvernement à l'époque. Du nom de Whitlam, premier ministre limogé pendant la crise constitutionnelle de 1975.

Living the life of Riley : menant une vie facile, en profitant du travail ou de l'argent de quelqu'un d'autre. Tiré d'un feuilleton radiophonique américain populaire dans les années quarante, qui donna lieu à un film.

being off with the pixies : se dit de quelqu'un qui rêvasse ou fuit la réalité, par exemple sous l'emprise de l'alcool.

eine kleine schnapbereft : invention de l'auteur, en allemand de cuisine - quelque chose comme « ça clackiert nicht » ou « une petite dose de déclaquant ».

\section{NOTES}

1. En français dans le texte.

2. En français dans le texte.

3. En français dans le texte. 


\section{AUTEURS}

\section{SHANE MALONEY}

Shane Maloney est un écrivain australien, auteur d'une série de romans policiers dont le héros, Murray Whelan, est un ancien attaché parlementaire devenu député travailliste. Ses romans, qui dépeignent le milieu politique de l'État de Victoria dans les années quatre-vingt-dix, sont écrits dans un style qui fait largement appel à l'argot et aux particularismes de l'anglais d'Australie. Son dernier livre en français est paru en 2006 aux éditions du Masque sous le titre Spécialité de fruits de mer (Something Fishy, traduction Serge Chwat et Pascale Michel). 